\title{
Articles of Significant Interest Selected from This Issue by the Editor
}

Behind Enemy Lines: Pathogen-Secreted Host Nucleus-Infiltrating Proteins (Infiltrins)

Many bacterial and protozoan pathogens have evolved subtle ways of subverting and escaping their host's protective immune responses, mainly by adopting an intracellular lifestyle. Parasitic worms, such as Schistosoma haematobium, are too large to hide inside cells. Pennington et al. (e00301-17) describe how the parasite's eggs, by secreting a glycoprotein called H-IPSE, may gain access to the innermost compartment of host cells, the nucleus, after crossing several biological barriers, such as the cytoplasmic, nuclear, and possibly also endosomal membranes-a surprising property which led the authors to introduce the name infiltrins for this class of proteins.

Cathelicidin-2, a Host Defense Peptide That Controls Pseudomonas aeruginosaInduced Lung Inflammation In Vivo

Patients with cystic fibrosis (CF) or chronic obstructive pulmonary disease (COPD) can suffer from recurrent infections with Pseudomonas aeruginosa that result in excessive inflammation and tissue damage. As antibiotic resistance complicates treatment of these recurrent infections, antimicrobial peptide (AMP)-based therapies have been suggested as new alternatives. Coorens et al. (e00546-17) show that the AMP chicken cathelicidin-2 (CATH-2) not only kills $P$. aeruginosa but also controls $P$. aeruginosainduced lung inflammation in vivo. This evidence further strengthens the case for developing AMP-based anti-infectives with both antimicrobial and immunomodulatory functions and shows their potential as anti-infectives for CF and COPD patients.

Highly Virulent Aspergillus fumigatus Isolates Induce an Interleukin $1 \alpha$ Dependent Inflammatory Response

Although different Aspergillus fumigatus isolates are known to induce different inflammatory responses, the mechanism(s) behind how specific inflammatory responses are induced is not understood. Caffrey-Carr et al. (e00661-17) reveal a role for interleukin $1 \alpha$ $(\mathrm{IL}-1 \alpha)$ in the immune response specifically directed against highly virulent $A$. fumigatus isolates. The induction of IL- $1 \alpha$-dependent host resistance corresponded with the ability of isolates to germinate rapidly within the pulmonary environment. Moreover, fungal hyphae of isolates were sufficient to induce IL- $1 \alpha$-dependent immunity. These data demonstrate that fungal growth at the site of infection dictates the innate immune response necessary for maintaining host resistance.

\section{Zinc Promotes Intracellular Survival of Salmonella enterica Serovar} Typhimurium by Subverting Innate Immune Responses

Divalent metals play important roles in the host-pathogen interaction either by acting as essential nutrients for microbes or by modulating both microbial and host defense mechanisms. Wu et al. (e00418-17) demonstrate that the intracellular bacterium Salmonella enterica serovar Typhimurium mobilizes zinc to block antimicrobial effector pathways of macrophages, including the formation of nitric oxide or reactive oxygen species. This effect of zinc is based on inhibition of NF- $\kappa$ B-mediated transcriptional activation of innate immune genes. Accordingly, zinc chelation or overexpression of zinc-binding metallothioneins by macrophages results in improved control of Salmonella infection. 